\title{
Lexis
}

Journal in English Lexicology

Book reviews | 2010

Laure LANSARI, Linguistique contrastive et traduction. Les périphrases verbales aller + infinitif et be going to Éditions Ophrys, 2009, 258 pages

\section{Lucile Bordet}

\section{OpenEdition}

1 Journals

Édition électronique

URL : http://journals.openedition.org/lexis/1775

DOI : $10.4000 /$ lexis. 1775

ISSN : 1951-6215

\section{Éditeur}

Université Jean Moulin - Lyon 3

\section{Référence électronique}

Lucile Bordet, "Laure LANSARI, Linguistique contrastive et traduction. Les périphrases verbales aller + infinitif et be going to », Lexis [En ligne], Recensions, mis en ligne le 06 juin 2010, consulté le 24 septembre 2020. URL : http://journals.openedition.org/lexis/1775; DOl : https://doi.org/10.4000/lexis. 1775

Ce document a été généré automatiquement le 24 septembre 2020.

\section{$\Theta \Theta \Theta \Theta$}

Lexis is licensed under a Creative Commons Attribution-NonCommercial-NoDerivatives 4.0 International License. 


\title{
Laure LANSARI, Linguistique contrastive et traduction. Les périphrases verbales aller + infinitif et be going to
}

Éditions Ophrys, 2009, 258 pages

\author{
Lucile Bordet
}

\section{RÉFÉRENCE}

Laure Lansari

Linguistique contrastive et traduction. Les périphrases verbales aller + infinitif et be going to.

Éditions Ophrys, 2009. ISBN : 978-2-7080-1248-6, Prix : $35 €, 258$ pages

1 L'ouvrage s'ouvre sur une introduction de quatre pages dans laquelle l'auteur présente les raisons qui l'ont conduite à mener cette étude contrastive sur aller + infinitif et be going to en français et en anglais contemporains. Elle revient sur la "comparatibilité » des deux formes qui en a motivé le rapprochement et note une "convergence formelle » (p.1). En effet, les deux formes périphrastiques se fondent sur un même processus de métaphorisation qui conduit un verbe dénotant initialement un déplacement spatial à être utilisé pour renvoyer à un déplacement temporel, à savoir l'intention ou le futur proche. L'auteur souligne également un "parallélisme fonctionnel, dans la mesure où [les deux structures] expriment toutes deux l'intention et le futur proche selon les grammaires traditionnelles » (p. 1).

2 L'auteur indique que les données diachroniques dont elle dispose montrent que les périphrases ont toutes deux connu une expansion à la fin du Moyen-âge. Elle s'appuie sur les travaux de J.-P. Vinay et J. Darbelnet (1958), d'A.Celle $(1997,2006)$ et de P. Larreya $(2001,2005)$ pour démontrer que malgré leurs ressemblances, aller + infinitif et be going to présentent pourtant de nombreuses divergences d'emploi. Ce sont ces différences que L. Lansari se propose d'étudier dans le cadre de la Théorie des 
Opérations Enonciatives (T.O.E.) tout au long de cet ouvrage, qui se subdivise en cinq chapitres.

3 L'auteur explique son choix de travailler essentiellement sur un corpus de textes traduits. Ceux-ci facilitent, selon elle, la mise en évidence de phénomènes plus problématiques à déceler dans des textes originaux, mais elle ne précise pas lesquels. Le corpus analysé se compose de 312 énoncés anglais > français et 465 énoncés français > anglais extraits de textes littéraires et journalistiques exclusivement. Les exemples ont été majoritairement tirés du corpus informatisé PLECI et d'un relevé manuel. Les grammaires traditionnelles considèrent les tournures périphrastiques plus fréquentes dans un registre peu soutenu, plus caractéristique de l'oral que de l'écrit. Elle déplore donc l'absence de corpus oral, mais explique que rassembler des données orales traduites est trop complexe. Cela aurait appelé à mener une étude différentielle de be going to et gonna du point de vue de leur grammaticalisation, ce qui dépasse la perspective contrastive centrée sur la traduction adoptée dans cet ouvrage.

4 Le chapitre I de 14 pages intitulé «Les périphrases dans les travaux typologiques: questions théoriques » revient sur les problèmes que soulève la typologie linguistique. L'auteur rappelle brièvement que «la typologie linguistique cherche à dégager des universaux de langage » (p.5). Elle se fonde sur les travaux de B. Heine et T. Kuteva (2002), pour expliquer la grammaticalisation des verbes de déplacement spatial en verbes de déplacement temporel par le truchement d'un processus de métaphorisation. L'auteur rejette cependant l'hypothèse métaphorique qui s'avère selon elle problématique pour l'étude contrastive qu'elle se propose de conduire. Pour l'auteur, on ne peut postuler une " équivalence translangagière » simpliste ; il est nécessaire de prendre en considération les données contextuelles, comme le veulent les études contrastives. S'appuyant sur la T.O.E., l'auteur indique qu'elle a choisi d'adopter une démarche sémasiologique, seule démarche capable de rendre compte des divers emplois d'aller + infinitif et de be going to.

5 L. Lansari s'interroge ensuite sur l'équivalence sémantique des lexèmes go et aller et indique que les partisans de la T.O.E. ignorent généralement le sémantisme de ces deux lexèmes. Définir un invariant suppose que l'on ne recoure qu'à une métalangue abstraite, même pour rendre compte des emplois spatiaux de go et aller; elle choisit donc d'écarter la notion de déplacement spatial de son analyse. Sur le plan morphosyntaxique, l'auteur démontre que l'équivalence entre les deux formes ne saurait être que partielle car elles n'ont pas le même paradigme. Ainsi, l'emploi de la structure aller + infinitif ne saurait être l'équivalent de : (13) 'Messenger...'

'What ?' Helen does not answer, but goes red and looks as if she might be going to cry. (Lodge1, 281)

qui serait plutôt traduit par

(13') - Messenger...

Quoi? » en guise de réponse, Helen rougit et paraît sur le point de fondre en larme.

que par aller + infinitif.

6 L. Lansari avance alors l'hypothèse « que la correspondance établie entre aller + inf. et be going to, n'est finalement qu'une correspondance de surface, qui cache des opérations sous-jacentes divergentes » (p.14) et renouvelle son intention de mettre au jour les contextes permettant d'établir l'équivalence entre les deux formes mais également de s'attarder sur les éléments contextuels qui mettent à mal cette "correspondance de surface ». 
L'auteur propose ensuite une analyse critique des travaux sur la grammaticalisation qui invoquent, selon elle, trop souvent le phénomène de métaphorisation et plus récemment le processus métonymique en guise de justification de la diffusion des « gofutures ». À l'explication métaphorique, et au postulat métonymique, elle favorise l'hypothèse allative, c'est-à-dire le déplacement vers un but.

7 Le chapitre II, constitué de 30 pages et intitulé «État de la question ", synthétise de manière argumentée les travaux antérieurs sur les deux structures périphrastiques. Ce chapitre retrace le développement diachronique des deux périphrases avant de proposer quelques hypothèses en synchronie. Pour aller + infinitif, l'auteur s'appuie sur les travaux de G. Gougenheim ([1929] 1971: 92) qui distingue un effet de sens inchoatif dès le XIVe ainsi qu'un effet de sens de "futur prochain" plus tardif au XVe notamment dans les pièces de théâtre. Quant à be going to, elle constate des "données plus lacunaires » (p. 22). La construction anglaise aurait connu une concurrence avec go to, maintenant archaïque. Elle développe "l'hypothèse du contact» avec une périphrase française au XVIe, hypothèse qu'elle juge peu satisfaisante.

En synchronie, l'auteur revient tout d'abord sur le registre de langue et les supports les plus propices à l'utilisation des périphrases. Elle note que le registre familier en favorise l'apparition tant à l'écrit qu'à l'oral et tire une première conclusion qui différencie la périphrase anglaise de son «équivalent» français: si l'on retrouve be going to presque exclusivement dans les dialogues et passages narratifs de fiction, aller + inf. connaît une utilisation plus libre (oral, écrit, textes fictionnels, informatifs, etc.). L'auteur procède ensuite à un examen critique des deux effets de sens généralement mis en avant, à savoir le futur proche et l'intention. Elle rappelle que l'invariant de ces constructions ne saurait se réduire à l'expression du futur proche ou de l'intention et insiste sur l'importance des facteurs situationnels et contextuels. Elle procède alors à une distinction entre « intention » et " volonté » et offre une critique de l'intention qui ne serait qu'un effet de sens possible selon la relation construite entre sujet et prédicat. A l'intention, qui serait selon les termes de P. Cotte « intraprédicative », elle préfère la " décision ", qui lui semble plus pertinente, dans la mesure où les périphrases étudiées permettent « la construction d'une relation entre le sujet énonciateur et l'ensemble de la relation prédicative à valider » (p. 31).

Elle rappelle ensuite brièvement les notions d'Intérieur, d'Extérieur et de Frontière propres à la T.O.E. et propose une redéfinition de l'intention, de la décision et de la volonté en termes d'opérations sur le domaine notionnel et de "construction de l'altérité ». Reprenant les travaux de J.-J. Franckel et D. Lebaud (1990), l'auteur développe la notion d'« intentionnalité » comme perception de l'Intérieur du domaine notionnel en tant que place nécessaire du sujet, et l'oppose à la notion d'« intention » précédemment définie dont elle rappelle qu'elle est à envisager comme une " modalité radicale reposant sur une élimination de l'altérité»(p.36). Elle applique cette définition d'intentionnalité à aller + inf. et be going to et conclut à la même construction de l'altérité pour les deux constructions avant de s'interroger sur le statut des formes périphrastiques dans la T.O.E. Elle rappelle que les formes périphrastiques « relèvent par définition de l'aspect grammatical» (p.39) dans la T.O.E., mais que les études tendent plutôt à associer be going to à la modalité. Elle adopte une position plus modérée et précise que selon les contextes les périphrases pourront revêtir des valeurs modales et/ou aspectuelles. 
10 Si l'auteur ne l'a pas explicitement mentionné, ce chapitre, qui semblait pourtant avoir vocation à dégager un invariant pour ces deux constructions, se clôt en ne proposant pas de réel invariant pour les deux formes. Toutefois, grâce à son appareil critique des théories existantes qui étudient les valeurs de aller + inf et be going to il a le mérite de poser le cadre théorique en discutant et en affinant les valeurs souvent considérées comme généralement admises. C'est plus tard dans le développement que l'auteur dégagera un invariant aux deux constructions.

11 Les chapitres III et IV, respectivement composés de 61 et 72 pages, proposent une analyse du corpus littéraire et montrent que l'équivalence entre les deux structures n'est possible que dans ce type de textes.

12 Le chapitre III, intitulé « Correspondance entre aller + inf. et be going to dans le corpus littéraire ", s'attache plus particulièrement à mettre au jour les paramètres permettant la traduction d'une périphrase par l'autre en dépit des divergences fondamentales relevées entre les deux constructions. L'auteur commence par une présentation de son corpus littéraire et en reconnaît les limites du point de vue de la représentativité en raison du nombre restreint d'énoncés étudiés. Reprenant les travaux de P. Larreya (2001) et d'A. Celle (1997), elle aborde deux points qui lui semblent permettre de rendre compte des points de convergence et de divergence entre les deux périphrases. Selon elle, il faut tenir compte du type de repérage et de la référence spécifique ou générique de l'énoncé. Elle présente celle-ci en termes de détermination quantitative (Qnt) et qualitative (Qlt) de la notion, selon la tradition de la T.O.E. Elle explore les notions d'« autonomie » et d'« implication » pour différencier will et be going to mais finit par les écarter pour mieux recentrer son argumentation sur la notion de "préconstruction ", subdivisée en « préconstruction existentielle » et « préconstruction discursive », qu'elle juge plus apte à effectuer une telle distinction et qu'elle pose même comme invariant à aller + inf. et be going to : «La préconstruction du choix de I (sans mention de E) nous semble une condition nécessaire à la correspondance entre aller + inf. et be going to. (...) Il reste à savoir, comment, à partir de cet invariant général, émergent diverses valeurs ou effets de sens»(p.67). L'auteur organise son développement selon les types d'énoncés (assertifs, négatifs, etc.) et le type de personne utilisé comme sujet syntaxique et montre leur influence sur les valeurs que vont revêtir les périphrases (intention, décision, etc.).

13 Au terme d'une analyse détaillée du corpus littéraire dont elle a reproduit de nombreux exemples, analyse qui mêle le point de vue énonciatif au point de vue contrastif, l'auteur dégage quelques points de convergence entre les deux constructions :

- be going to et aller + inf. seraient caractérisés par une opération de préconstruction, « une non prise en compte de l'altérité » (p.111) (entendez que l'on n'envisage pas la validation de $\mathrm{E}$, mais uniquement celle de I).

- Lorsque les constructions renvoient à l'avenir, elles prennent soit une valeur d'intention / de décision, soit une valeur d'inférence.

Néanmoins, elle souligne d'une part que ces convergences sont minoritaires par rapport aux divergences d'emploi et d'autre part, qu'au sein même des apparentes correspondances entre les deux structures résident des subtilités d'emploi qui ne permettent pas de les considérer comme véritablement équivalentes, sauf données contextuelles particulières.

15 Ce sont justement ces nombreuses divergences qu'elle se propose d'étudier en détail dans le chapitre IV intitulé «Déstabilisation de la correspondance dans le corpus 
littéraire ». Il se divise en deux grandes sections : l'une consacrée aux traductions d' aller + infinitif et l'autre aux traductions possibles de be going to. Dans le sens français > anglais, on remarquera une tendance à la modalisation, ainsi qu'une tendance - plus marginale - à l'aspectualisation avec des structures du type be about to. L'auteur souligne que les traductions d'aller + inf. relèvent de la tendance à «l'explicitation modale » en anglais (p.112), will étant la traduction la plus fréquente. Elle explore ensuite les traductions en shall dans des contextes permettant ce qu'elle appelle « la mise en retrait de l'énonciateur " (p. 126), mais aussi des traductions injonctives du type base verbale nue ou Let me / 's + BV qui s'inscrivent dans une relation intersubjective et correspondent à un évitement de l'assertion. Elle étudie également des traductions plus marginales du type be to, mais rappelle que celles-ci sont plus fréquentes dans le corpus journalistique. Dans le sens anglais $>$ français, on retrouve une tendance non plus à l'aspectualisation, mais à la temporalisation avec des traductions de be going to par des futurs ou des conditionnels, ainsi qu'une tendance à la modalisation (généralement radicale) par le biais de lexèmes tels pouvoir, vouloir, devoir, etc. Au terme de l'analyse d'exemples pertinents choisis dans le corpus, Laure Lansari conclut qu'aller + infinitif est déterminé qualitativement et ne fait pas l'objet d'une préconstruction contrairement à be going to qui peut marquer une détermination quantitative, et dont l'utilisation est conditionnée par une opération de préconstruction préalable.

Quant au chapitre $\mathrm{V}$, il propose une étude des textes journalistiques du corpus. Il s'ouvre sur un premier constat : la périphrase anglaise est quatre fois moins représentée dans le corpus journalistique que la périphrase française. L'auteur souligne que l'équivalence aller + inf. > be going to est mise à mal par le corpus journalistique car seules $9 \%$ des énoncés présentent des traductions d'une périphrase par l'autre (contre $38 \%$ dans le corpus littéraire). On apprend également que la proportion de traductions d'aller + infinitif par will demeure stable entre les deux corpus. L'auteur offre tout d'abord une définition du genre journalistique et caractérise celui-ci par une modalisation minimale, un effacement de la subjectivité et une mise en retrait de la détermination qualitative.

Le chapitre $\mathrm{V}$ s'attache à dégager les raisons de la quasi-absence de be going to au profit d'une traduction par un verbe lexical au prétérit dans le corpus journalistique. Elle démontre que dans ce type de texte, le renvoi à l'avenir se construit grâce à un effacement du sujet. Or, selon l'auteur : « en tant que marqueur d'inférence, [be going to] semble nécessiter un ancrage situationnel fort, incompatible avec l'effacement de la source assertive » (p.194). Est enfin abordée la notion de rétrospection accompagnée d'une étude détaillée des différents marqueurs présents dans les traductions, comme les adverbes, le type de procès décrit, et les opérations d'agencement ou de réagencement syntaxique. L. Lansari démontre que la rétrospection est une opération subjective et la seconde cause de la faible fréquence d'utilisation de be going to.

18 Le présent ouvrage, original par l'approche choisie et la problématique traitée, permet à l'auteur, par le biais d'une analyse contrastive et énonciative, de montrer que l'équivalence généralement admise en aller + infinitif et be going to est loin d'être systématique. Elle ne saurait donc être le reflet d'une véritable équivalence translangagière. Il serait alors préférable de postuler une «correspondance contextuelle, nécessairement partielle » (p. 239). 

cela fasse l'objet d'une étude future. Cependant, cet ouvrage ne manquera pas d'apporter un précieux éclairage théorique pour tous les lecteurs qui s'intéressent à la traduction, aux structures périphrastiques et plus largement à la T.O.E.

\section{BIBLIOGRAPHIE}

CELLE A., Étude contrastive du futur français et de ses réalisations en anglais, Paris, Gap, Ophrys, 1997.

CHUQUET H. \& PAILLARD M., Approche linguistique des problèmes de traduction anglais - français, Paris, Gap, Ophrys, 1987.

CULIOLI A., Pour une linguistique de l'énonciation 1, 2, 3, Paris, Gap, Ophrys, 1990, 1999.

FRANCKEL J.-J., Étude de quelques marqueurs aspectuels du français, Paris, Genève, Droz, 1989.

GROUSSIER M.-L. et RIVIÈRE C., Les mots de la linguistique. Lexique de linguistique énonciative, Paris, Gap, Ophrys, 1996.

HOPPER P. J. \& TRAUGOTT E.C., Grammaticalization, Cambridge, Cambridge University Press, 1993.

\section{AUTEURS}

\section{LUCILE BORDET}

Lucile Bordet, Centre d'Études Linguistiques, Université Jean Moulin - Lyon 3. 
Lucile Bordet est PrAg en Linguistique anglaise dans le Département d'Études Anglophones de l'Université Jean Moulin - Lyon 3, en France. Elle travaille actuellement à la rédaction d'une thèse sur l'évolution phonologique, syntaxique et fonctionnelle de HAVE, BE et DO du point de vue de la grammaticalisation. 